\title{
Predictive value of gamma-glutamyl transpeptidase to lymphocyte count ratio in hepatocellular carcinoma patients with microvascular invasion
}

Hongxing Zhang ${ }^{1 \dagger}$, Yu Zhou ${ }^{1+}$, Yicheng Li ${ }^{1,2 \dagger}$, Wanying Qin ${ }^{1}$, Yunhua Zi ${ }^{1}$, Yulan Liu ${ }^{1}$, Xiaoying Qiu ${ }^{1}$, Hongyuan Xu', Weijia Liao ${ }^{1 *}$ (D) and Zhaoquan Huang ${ }^{1,3^{*}}$

\begin{abstract}
Background: Microvascular invasion (MVI) is an independent risk factor for poor prognosis in hepatocellular carcinoma (HCC). However, there is still a lack of preoperative markers to predict MVI in HCC. This study intends to explore the potential application value of the gamma-glutamyl transpeptidase (GGT) to lymphocyte count ratio (GLR) in predicting MVI in HCC and provide guidance for clinical diagnosis and treatment.

Methods: From March 2010 to December 2015, 230 HCC patients who underwent surgical treatment in the Affiliated Hospital of Guilin Medical University were selected. Clinicopathological parameters between the MVI group $(n=115)$ and the non-MVI group ( $n=115)$ were comparatively analyzed. The GLR was used as the potential risk factor for HCC with MVI, and its optimal cut-off value was estimated by using the receiver operating characteristic (ROC) curve. The Kaplan-Meier method was used to analyze the survival of HCC patients, and univariate and multivariate Cox regression analyses were used to establish independent predictors affecting postoperative HCC patients.

Results: The GLR levels in the MVI group and non-MVI group were $84.83 \pm 61.84$ and $38.42 \pm 33.52(p<0.001)$, respectively. According to ROC curve analysis, the optimal cut-off value of GLR was 56.0, and the area under the ROC curve (AUC) was 0.781 (95\% Cl, 0.719-0.833) for the risk prediction of MVI in HCC patients. Multivariate analysis showed that tumor size $>5 \mathrm{~cm}$, HCC combined with MVI and GLR > 56.0 were independent risk factors for poor prognosis in HCC patients. In addition, compared with the non-MVI group, patients in the MVI group had shorter progression-free survival (PFS) and overall survival (OS).
\end{abstract}

Conclusion: GLR could be a predictive biomarker of HCC after operation and a potential predictor of HCC combined with MVI.

Keywords: Hepatocellular carcinoma, Microvascular invasion, GLR, Predictive

\footnotetext{
* Correspondence: liaoweijia288@163.com; huang788766@163.com

${ }^{+}$Hongxing Zhang, Yu Zhou and Yicheng Li contributed equally to this work.

'Laboratory of Hepatobiliary and Pancreatic Surgery, Affiliated Hospital of

Guilin Medical University, Guilin 541001, Guangxi, People's Republic of China

Full list of author information is available at the end of the article
}

(c) The Author(s). 2020 Open Access This article is distributed under the terms of the Creative Commons Attribution 4.0 International License (http://creativecommons.org/licenses/by/4.0/), which permits unrestricted use, distribution, and reproduction in any medium, provided you give appropriate credit to the original author(s) and the source, provide a link to the Creative Commons license, and indicate if changes were made. The Creative Commons Public Domain Dedication waiver (http://creativecommons.org/publicdomain/zero/1.0/) applies to the data made available in this article, unless otherwise stated. 


\section{Background}

Hepatocellular carcinoma ( $\mathrm{HCC}$ ) is the most common primary liver malignancy. According to the global cancer statistics in 2018, the number of new cases of liver cancer reached 841,080, and there were 781,631 deaths [1]. Liver cancer is a significant public health problem worldwide, especially in China [2]. Studies have shown that MVI is a considerable risk factor for poor postoperative prognosis in HCC, MVI leads to early postoperative recurrence and metastasis and is an independent predictor of long-term postoperative survival [3, 4]. The number of MVI, depth of infiltration, and distance of invasion all affect the prognosis of postoperative HCC patients $[5,6]$. In recent years, there have been many models for the diagnosis, treatment and prognosis of HCC [7, 8]. Therefore, the use of models to assess whether patients have preoperative $\mathrm{HCC}$ metastasis or HCC combined with MVI has tremendous clinical significance for selecting appropriate individualized treatment methods and improving the prognosis and survival of HCC patients.

Studies have shown that tumors are malignant transformations stimulated by long-term inflammatory factors, with inflammation as the driving factor and cancer as the result [9]. Inflammatory cytokines are essential participants in regulating the tumor microenvironment, and they can promote the proliferation and survival of tumor cells and enhance angiogenesis, invasion and metastasis [10]. Chronic inflammation and inflammationrelated factors are the premise or basis of tumorigenesis. However, what are the vital inflammatory factors? There is no conclusion yet. Therefore, it is necessary to explore and identify the related inflammatory factors affecting the occurrence and progression of tumors to assist clinical diagnosis and treatment and achieve the fundamental purpose of the treatment or prevention of tumors. Recent studies have found that the GLR index plays an essential role in tumor progression, and its prognostic potential is superior to that of other inflammatory scoring systems [11]. It is speculated that GLR may be a critical factor in the occurrence of HCC combined with MVI and affect the survival and prognosis of HCC patients.

This study aims to explore the potential value of GLR in predicting the risk of HCC with MVI and its significance in predicting the prognosis of $\mathrm{HCC}$ to provide a new basis for the development of clinical treatment plans.

\section{Material and methods}

\section{Patients}

In this article, 230 patients with hepatocellular carcinoma (115 in the MVI group and 115 in the non-MVI group) who underwent surgical treatment at the
Affiliated Hospital of Guilin Medical University were selected. All patients were diagnosed by clinical examination, ultrasonography (US), magnetic resonance imaging (MRI), thoracic and abdominal CT, angiography and hematology. The resected samples of all patients were confirmed by pathological examination. In this paper, according to the study by Sumie et al. [12], we defined the group in which MVI was not found as the non-MVI group, the group in which 1-5 MVIs was found as the M1 group, and the group in which more than 5 MVIs were found as the M2 group. Among the MVI patients, 70 patients were in the M1 group, and 45 patients were in the M2 group. Table 1 lists the clinicopathological parameters of the patients, such as demographic characteristics (age, sex, life history), history of hepatitis virus infection, hematological examination parameters (blood routine, liver function, protein levels, bilirubin, alphafetoprotein (AFP), GLR, etc.), and the characteristics of the tumor (the degree of cirrhosis, size and number). This study conformed to the Declaration of Helsinki and was approved by the research ethics committee of the Affiliated Hospital of Guilin Medical University. Written informed consent was obtained from all patients.

\section{Surveillance after hepatic resection}

Based on the inclusion and exclusion criteria, a total of 230 patients with liver cancer who underwent radical resection entered the study. Patients were excluded if they had: (1) patients whose pathological diagnosis was not liver cancer (HCC), such as cholangiocarcinoma (CCC); (2) patients who died during the perioperative period; (3) patients with incomplete data or lost contact during the follow-up period; (4) infectious disease, immune system disease, blood system disease, or use of drugs that affect blood within 1 month; (5) patients underwent arterial chemoembolization before surgery or Radiofrequency ablation; (6) HIV positive patients. The contents and requirements of periodical follow-up, please refer to our previous and relevant reports $[13,14]$. Progression-free survival (PFS) was defined as the period from the date of surgery to the date of metastasis, recurrence, death, or the last date on which the disease activity was evaluated, while overall survival (OS) was defined as the period from the date of surgery to the date of death or the last follow-up.

\section{Ascertainment of the cut-off value of GLR}

To assess the risk of HCC combined with MVI, we analyzed the receiver operating characteristic (ROC) curve to determine the optimal cut-off value of preoperative GLR, which should have relatively high sensitivity and 
Table 1 Clinical and biochemical data of examined patients

\begin{tabular}{|c|c|c|c|}
\hline \multirow[t]{2}{*}{ Parameter } & \multirow{2}{*}{$\begin{array}{l}\text { Non MVI group } \\
\text { Mean } \pm \mathrm{SD}^{*}(n=115)\end{array}$} & \multirow{2}{*}{$\begin{array}{l}\text { MVI group } \\
\text { Mean } \pm \mathrm{SD}^{\mathrm{a}}(\mathrm{n}=115)\end{array}$} & \multirow[t]{2}{*}{$p$ value } \\
\hline & & & \\
\hline Sex: female / male (n) & $21 / 94$ & $9 / 106$ & 0.019 \\
\hline Age (years) & $50.23 \pm 12.28$ & $48.34 \pm 11.00$ & 0.221 \\
\hline Drinking: absent / present (n) & $57 / 58$ & $52 / 63$ & 0.509 \\
\hline Smoking: absent / present (n) & $73 / 42$ & $66 / 49$ & 0.345 \\
\hline HBsAg: negative / positive ( $\mathrm{n}$ ) & $27 / 88$ & $17 / 98$ & 0.094 \\
\hline HCVAb: negative / positive ( $\mathrm{n}$ ) & $109 / 6$ & $113 / 2$ & 0.280 \\
\hline Family history: absent/present (n) & $99 / 16$ & $95 / 20$ & 0.468 \\
\hline Tumor number: single/multiple (n) & $86 / 29$ & $72 / 43$ & 0.047 \\
\hline Tumor size $(\mathrm{cm})$ & $6.08 \pm 3.32$ & $8.53 \pm 3.78$ & $<0.001$ \\
\hline Liver cirrhosis: absent / present (n) & $9 / 106$ & $7 / 108$ & 0.796 \\
\hline $\operatorname{NEUT}\left(\times 10^{9} / \mathrm{L}\right)$ & $3.71 \pm 1.50$ & $4.52 \pm 2.24$ & 0.001 \\
\hline LYMPH $\left(\times 10^{9} / \mathrm{L}\right)$ & $1.77 \pm 0.61$ & $1.58 \pm 0.59$ & 0.012 \\
\hline WBC $\left(\times 10^{9} / L\right)$ & $6.17 \pm 1.85$ & $6.87 \pm 2.50$ & 0.017 \\
\hline Platelets $\left(\times 10^{9} / \mathrm{L}\right)$ & $172.43 \pm 72.73$ & $180.14 \pm 82.65$ & 0.453 \\
\hline Albumin (g/L) & $41.32 \pm 4.01$ & $39.47 \pm 4.62$ & 0.002 \\
\hline Globulin (g/L) & $29.83 \pm 4.79$ & $31.34 \pm 6.52$ & 0.045 \\
\hline TBIL $(\mu \mathrm{mol} / \mathrm{L})$ & $13.22 \pm 4.93$ & $15.07 \pm 12.61$ & 0.146 \\
\hline $\mathrm{DBIL}(\mu \mathrm{mol} / \mathrm{L})$ & $4.47 \pm 2.32$ & $5.48 \pm 4.10$ & 0.022 \\
\hline $\mathrm{ALT}(\mathrm{U} / \mathrm{L})$ & $37.38 \pm 30.49$ & $44.05 \pm 33.32$ & 0.115 \\
\hline AST (U/L) & $39.33 \pm 30.06$ & $55.15 \pm 35.29$ & $<0.001$ \\
\hline ALP $(U / L)$ & $81.45 \pm 87.65$ & $102.22 \pm 50.98$ & 0.030 \\
\hline GGT (U/L) & $59.17 \pm 40.61$ & $114.51 \pm 64.46$ & $<0.001$ \\
\hline AFP (ng/ml): median, range & $44.60,1.18-24,200$ & $457.80,0.61-25,410$ & 0.271 \\
\hline GLR level & $38.42 \pm 33.52$ & $84.83 \pm 61.84$ & $<0.001$ \\
\hline
\end{tabular}

${ }^{\mathrm{a}}$ Data presented as mean \pm SD or proportions

$n$ number of patients; $H B s A g$ hepatitis B surface antigen, HCVAb hepatitis $C$ virus antibody, NEUT neutrophil cell count, $L Y M P H$ lymphocyte count, WBC white blood cell, $T B I L$ total bilirubin, DBIL direct bilirubin, $A L T$ alanine aminotransferase, $A S T$ aspartate aminotransferase, $A L P$ alkaline phosphatase, GGT gamma-glutamyl

transpeptidase, AFP alpha-fetoprotein, GLR GGT to lymphocyte ratio

specificity. The other clinicopathological data were dichotomized: sex (male vs. female), age (> 50 age vs. $\leq 50$ age), drinking (present vs. absent), HBsAg (positive vs. negative), tumor number (multiple vs. single), tumor size $(>5 \mathrm{~cm}$ vs. $\leq 5 \mathrm{~cm}$ ), liver cirrhosis (present vs. absent), MVI type (MVI vs. non-MVI) and AFP level $(>20 \mathrm{ng} / \mathrm{ml}$ vs. $\leq 20 \mathrm{ng} / \mathrm{ml})$.

\section{Statistical analysis}

All statistical analyses were performed using SPSS 21.0 and MedCalc 11.3.0. For the count data conforming to a normal distribution, the independent sample $t$ test was used for comparisons between the groups. For the data that did not conform to a normal distribution, a nonparametric test (Mann-Whitney $\mathrm{U}$ method) was used for group comparisons. The Pearson chi-square test was used to compare qualitative variables. The ROC curve and Youden index were used to select the optimal cut-off value of GLR; and the Kaplan-Meier method was used to obtain the survival curve. The log-rank test was used to study the differences among different groups. Then, the variables with $p<0.05$ were analyzed by multivariate analysis. A Cox proportional hazards regression model was performed to determine the independent prognostic factors, and $p<0.05$ was considered to indicate a significant difference.

\section{Results}

Correlation of MVI with the clinicopathological features of HCC patients, and GLR is more like an inflammatory factor

By comparative analysis of the clinicopathological parameters of the MVI group $(n=115)$ and non-MVI group ( $\mathrm{n}=115)$, it was found that the GLR levels in the MVI group and non-MVI group were $84.83 \pm 61.84$ and $38.42 \pm 33.52$, respectively $(p<0.001)$. In addition to the GLR level, the MVI group had larger tumor sizes and 
higher neutrophil cell count (NEUT), white blood cell (WBC), globulin, direct bilirubin (DBIL), aspartate aminotransferase (AST), alkaline phosphatase (ALP) and GGT levels (all $p<0.05$ ) than the non-MVI group. In contrast, the lymphocyte count (LYMPH) and albumin levels were lower in the MVI group than in the nonMVI group $(p<0.05)$ (Table 1). In addition, we found that there was a positive correlation between the GLR level and AST level $(r=0.347, p<0.001)$ (Fig. 1b). These results suggest that inflammatory factors, such as NEUT, AST and GLR etc., increase the risk of MVI in patients with HCC.

\section{GLR may be a potential predictor in HCC complicated with MVI}

The ROC curve was drawn and analyzed according to the existence of MVI in patients with HCC. The optimum cut-off value of GLR was 56.0, the area under the ROC curve (AUC) was 0.781, and the 95\% confidence interval $(95 \%$ CI) was $0.719-0.833$. The sensitivity and specificity were 63.6 and $81.7 \%$, respectively, when the cut-off value of GLR was 56.0 (Fig. 1a). The results suggest that GLR may be a potential predictor for HCC complicated with MVI. Additionally, in the following studies, we found that when the cut-off value of GLR was 56.0, GLR had potential application value in predicting the postoperative survival of patients who had HCC and who had HCC combined with MVI.

\section{Univariate analysis and multivariate cox regression} analysis indicated that GLR > 56.0 was an independent risk factor for postoperative HCC

In univariate analysis, GLR > 56.0 was found to be a risk factor for postoperative PFS ( $\mathrm{HR}=2.36,95 \% \mathrm{CI}$, $1.53-3.08, p<0.001)$ and $\mathrm{OS}(\mathrm{HR}=2.47,95 \% \mathrm{CI}$, $1.80-3.40, p<0.001)$. In addition to GLR $>56.0$, the adverse factors of postoperative OS and PFS included multiple tumor nodules, tumor size $>5 \mathrm{~cm}$, MVI and AFP > $20 \mathrm{ng} / \mathrm{ml}$. The statistically significant factors in univariate analysis were further analyzed by the Cox proportional hazards regression model for multivariate analysis. GLR $>56.0$ was an independent risk factor for postoperative PFS $(\mathrm{HR}=1.56$, 95\% CI, $1.18-2.36, p=0.017)$ and $\mathrm{OS}(\mathrm{HR}=1.63$, $95 \% \mathrm{CI}, 1.28-2.31, p=0.006)$. In addition, tumor size $>5 \mathrm{~cm}$ and combined MVI can be used as independent risk factors for poor PFS and OS in HCC patients (Table 2).

The value of MVI and GLR in postoperative survival and prognosis of patients with HCC

Kaplan-Meier analysis showed that the mean PFS and OS of the non-MVI group $(n=115)$ were 51.1 months and 59.3 months, and those of the MVI group ( $\mathrm{n}=$ 115) were 26.9 months and 34.5 months, respectively. The PFS rates of the non-MVI group at 1 year, 3 years and 5 years were also significantly higher than those of the MVI group $(73.6 \%$ vs. $59.4,58.7 \%$ vs. 25.9 and $49.3 \%$ vs. $13.1 \%$, respectively, all $p<0.001$ ) (Fig. 2a). Similarly, the OS rates of the patients in the non-MVI group at 1 year, 3 years and 5 years were significantly higher than those of the MVI group $(88.1 \%$ vs. $66.8,73.0 \%$ vs. 37.1 and $61.4 \%$ vs. $17.9 \%$, respectively, $p<0.001$ ) (Fig. $2 \mathrm{~b}$ ). In addition, we further assessed the postoperative survival and prognosis of the M1 $(n=70)$ and M2 $(n=45)$ subgroups in the MVI group. Compared with the M2 group patients, the M1 group patients had a longer PFS $(p=0.019)$ (Fig. 2c) and OS $(p=0.010)$ (Fig. 2d).

Of the $230 \mathrm{HCC}$ patients involved in this study, compared with the GLR $\leq 56.0$ group $(n=138)$, the GLR >

56.0 group $(n=92)$ had a shorter mean PFS (46.9 months vs 28.1 months, $p<0.001)$ and OS (55.8 months
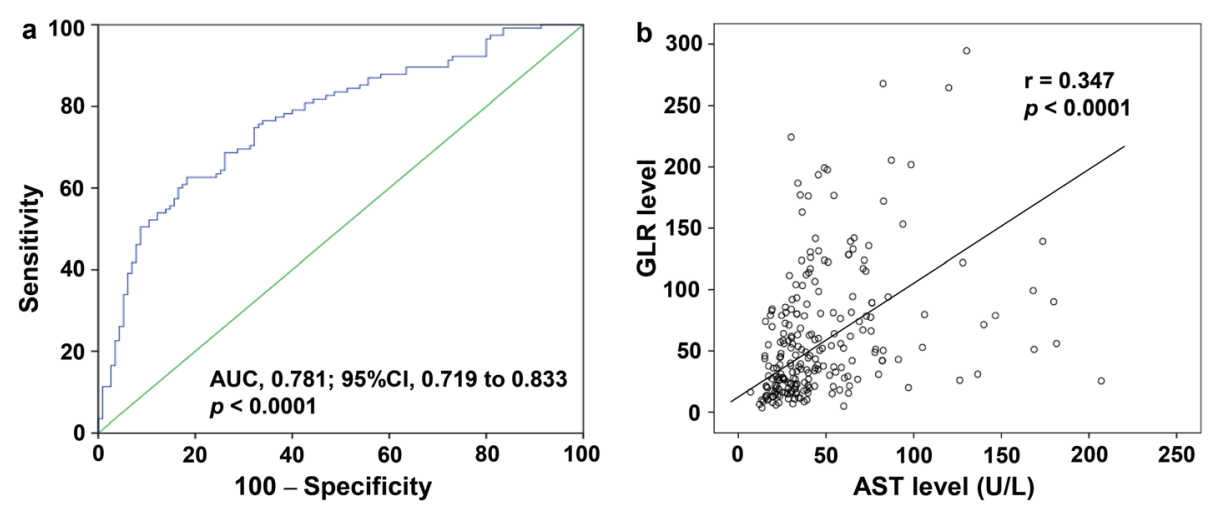

Fig. 1 Determination of the optimal GLR cut-off value and its correlation analysis with AST. a The ROC curve of GLR in patients with HCC; $\mathbf{b}$ The positive relation between the GLR level and AST level. MVI, microvascular invasion; GLR, gamma-glutamyl transpeptidase to lymphocyte ratio; AST, aspartate aminotransferase; ROC, receiver operating characteristic 
Table 2 Analysis predictors of progression-free survival and overall survival in patients with HCC

\begin{tabular}{|c|c|c|c|c|c|c|}
\hline \multirow[t]{2}{*}{ Variable } & \multicolumn{3}{|c|}{ Univariate analysis } & \multicolumn{3}{|c|}{ Multivariate analysis } \\
\hline & $\mathrm{HR}$ & $95 \% \mathrm{Cl}$ & $p$ value & $\mathrm{HR}$ & $95 \% \mathrm{Cl}$ & $p$ value \\
\hline \multicolumn{7}{|l|}{ Progression-free survival } \\
\hline Sex (male vs female) & 1.27 & $0.73-2.37$ & 0.507 & & & \\
\hline Age, y (> 50 vs $\leq 50)$ & 1.16 & $0.78-1.72$ & 0.446 & & & \\
\hline Drinking (present vs absent) & 0.95 & $0.64-1.42$ & 0.832 & & & \\
\hline HBsAg (positive vs negative) & 0.82 & $0.49-1.37$ & 0.469 & & & \\
\hline Tumor number (multiple vs single) & 1.78 & $1.16-2.80$ & 0.007 & & & \\
\hline Tumor size, cm (> 5 vs $\leq 5)$ & 2.61 & $1.73-3.37$ & $<0.001$ & 1.75 & $1.23-2.67$ & 0.003 \\
\hline Liver cirrhosis (present vs absent) & 1.33 & $0.65-2.75$ & 0.429 & & & \\
\hline MVI (MVI vs non-MVI) & 2.58 & $1.86-3.58$ & $<0.001$ & 1.91 & $1.35-2.71$ & $<0.001$ \\
\hline AFP, ng/ml (> 20 vs $\leq 20)$ & 1.59 & $1.12-2.26$ & 0.010 & & & \\
\hline GLR (> 56 vs $\leq 56)$ & 2.36 & $1.53-3.08$ & $<0.001$ & 1.56 & $1.18-2.36$ & 0.017 \\
\hline \multicolumn{7}{|l|}{ Overall survival } \\
\hline Sex (male vs female) & 1.13 & $0.62-2.09$ & 0.716 & & & \\
\hline Age, y (> 50 vs $\leq 50)$ & 1.08 & $0.73-1.61$ & 0.671 & & & \\
\hline Drinking (present vs absent) & 0.83 & $0.56-1.24$ & 0.371 & & & \\
\hline HBsAg (positive vs negative) & 0.72 & $0.43-1.21$ & 0.224 & & & \\
\hline Tumor number (multiple vs single) & 1.83 & $1.20-2.66$ & 0.004 & & & \\
\hline Tumor size, cm (> 5 vs $\leq 5)$ & 2.87 & $2.10-3.76$ & $<0.001$ & 2.11 & $1.48-3.07$ & $<0.001$ \\
\hline Liver cirrhosis (present vs absent) & 1.13 & $0.55-2.33$ & 0.732 & & & \\
\hline MVI (MVI vs non-MVI) & 2.83 & $2.05-3.61$ & $<0.001$ & 2.00 & $1.41-2.84$ & $<0.001$ \\
\hline AFP, ng/ml (> 20 vs $\leq 20)$ & 1.64 & $1.15-2.33$ & 0.006 & & & \\
\hline GLR (> 56 vs $\leq 56)$ & 2.47 & $1.80-3.40$ & $<0.001$ & 1.63 & $1.28-2.31$ & 0.006 \\
\hline
\end{tabular}

HR hazard ratio, $\mathrm{Cl}$ confidence interval, $H B s A g$ hepatitis B surface antigen, MVI microvascular invasion, AFP alpha-fetoprotein, GLR GGT to lymphocyte ratio

vs 33.4 months, $p<0.001$ ) (Fig. 3a, b). More interestingly, in the M1 group, patients $(\mathrm{n}=70)$ with GLR $>56.0$ $(n=38)$ had a shorter mean PFS (36.7 months vs 21.5 months, $p=0.012$ ) and mean OS (43.8 months vs 31.2 months, $p=0.031$ ) (Fig. 3c, d). This finding indicates that GLR can also play a prognostic role in the M1 group of HCC patients.

These results suggest that MVI (MVI subgroup) and GLR level are closely related to postoperative survival and the prognosis of patients with HCC after operation.

\section{Discussion}

The role of inflammatory factors in tumors has attracted much attention from researchers. Inflammation plays a decisive role in different stages of cancer development, including initiation, promotion, malignant transformation, invasion, and metastasis $[15,16]$. In the early stage of tumorigenesis, inflammatory cells can become influential tumor promoters, creating a favorable environment for tumor growth and promoting blood vessel growth [17-19]. Many factors have been found to be related to the occurrence of MVI in HCC; in this study, it is suggested that GLR is positively correlated with AST, and NEUT, AST, GLR and other inflammatory factors' level in the MVI group are higher than those in the nonMVI group, suggesting that these inflammatory factors may promote HCC progression and increase risk of MVI, which is in accordance with the researches which illuminate that anti-inflammatory treatment can effectively prevent early tumorigenesis and later malignant transformation [20, 21] .

In recent years, the construction of models for the diagnosis and prognosis prediction of hepatocellular carcinoma based on liquid biopsy has become a research hotspot [22-24]. The GLR prediction model built in this study was based on the ratio of GGT to lymphocyte count. Intrahepatic GGT mainly exists in the hepatocyte membrane and microsome and is a key enzyme in glutathione metabolism [25]; it plays an important role in hepatocarcinogenesis, vascular invasion and metastasis [26-28]. In addition, the overall survival rate of liver cancer patients with increased GGT is not favorable after liver tumor resection, radiofrequency ablation, and transcatheter arterial chemoembolization [29, 30]. Lymphocytes play a key role in cytotoxic cell apoptosis, inhibiting the production of inflammatory cytokines as well 

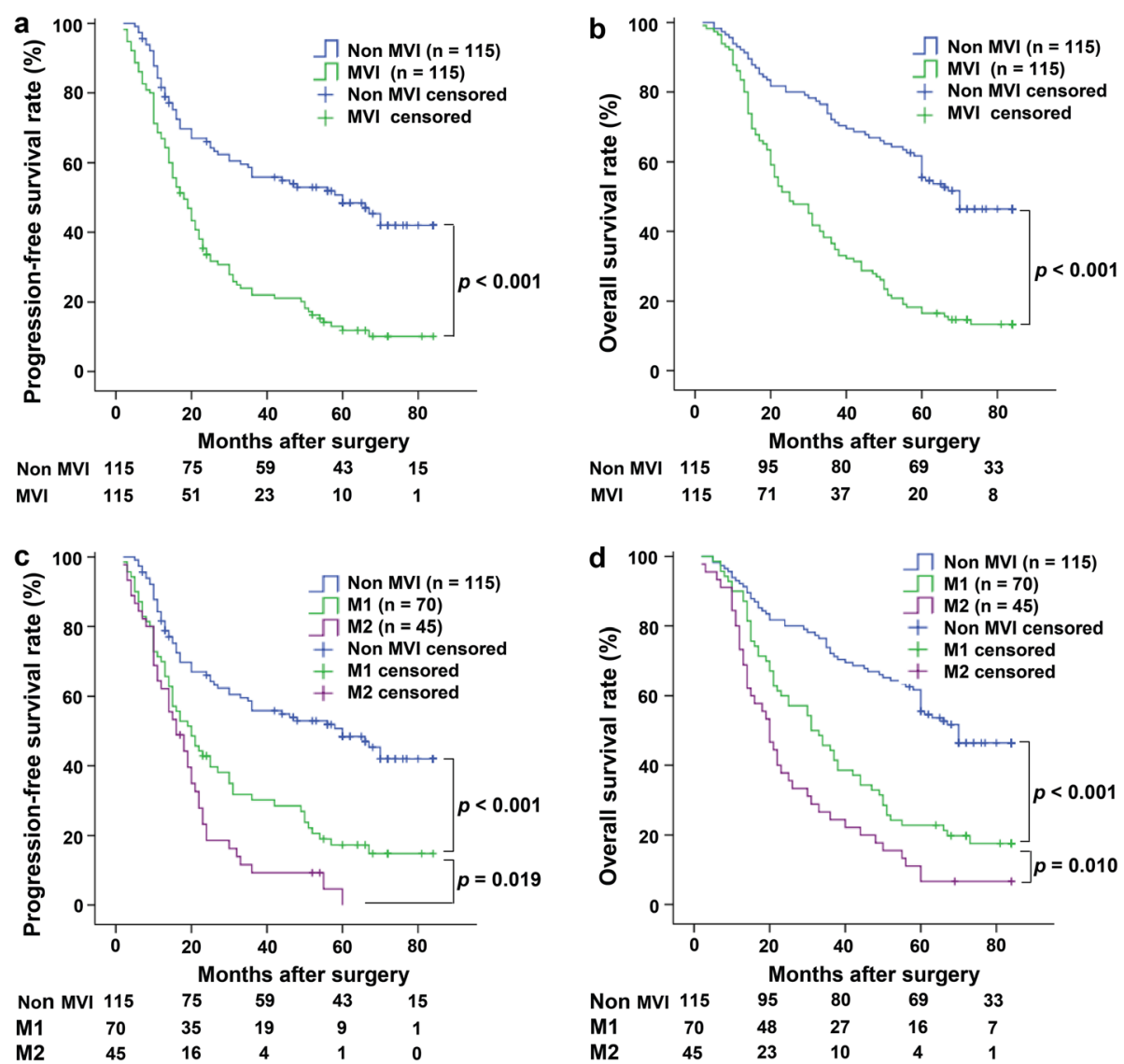

Fig. 2 The survival rate was analyzed based on the presence of MVI in HCC patients. a Compared with the non-MVI group, the MVI group had significantly worse survival $(p<0.001)$. b Compared with the non-MVI group, the OS of the MVI group tended to be worse $(p<0.001)$. $\mathbf{c}$ The PFS of the non-MVI patients was significantly higher than that of the M1 patients $(p<0.001)$; the PFS of the M1 patients was significantly higher than that of the M2 patients $(p=0.019)$. $\mathbf{d}$ The OS of the non-MVI patients was significantly higher than that of the M1 patients $(p<0.001)$, and the OS of the M1 patients was significantly higher than that of the M2 patients $(p=0.010)$

as the proliferation and migration of tumors in the body's anti-tumor immune response [31-33]. In this study, the prediction of the postoperative survival of patients with $\mathrm{HCC}$ and $\mathrm{HCC}$ complicated with MVI by GLR was assessed, and the results showed that GLR can provide an excellent individualized prediction ability for HCC patients combined with MVI after operation. Univariate analysis of follow-up data showed that multiple tumor nodules, tumor size $>5 \mathrm{~cm}$, MVI, AFP > $20 \mathrm{ng} / \mathrm{ml}$ and GLR > 56.0 were correlated with a shorter PFS and OS. Multivariate Cox regression analysis revealed that tumor size $>5 \mathrm{~cm}$, MVI and GLR > 56.0 were independent predictors of poor prognosis for HCC after operation. This result is consistent with previous research findings that tumor size is an independent predictor of OS in HCC patients [34]. Other previous studies also showed that MVI is characterized by vascular infiltration and invasive phenotypes and is associated with the poor prognosis of liver cancer [35]. The results of univariate and multivariate Cox regression analyses of the subjects in this study were consistent with those of the above studies, which further indicates that HCC with tumor size $>5 \mathrm{~cm}$ and combined with MVI has a higher degree of malignancy.

This study further found that patients in the non-MVI group had a better OS and PFS than those in the MVI group, while the OS and PFS of the HCC patients in the M1 group were better than those in the M2 group. These results suggest that it is feasible and necessary for MVI patients to receive active surgical treatment. Although the univariate analysis used in this study showed that multiple tumor nodules and AFP > $20 \mathrm{ng} / \mathrm{ml}$ were predictors of undesirable PFS and OS, none of these factors were identified as independent predictors in the multivariate analysis. However, this does not mean that there is no association between these factors and HCC prognosis and metastasis; they can also be used as potential prognostic factors for HCC patients after resection. For example, multiple or single tumor nodules 

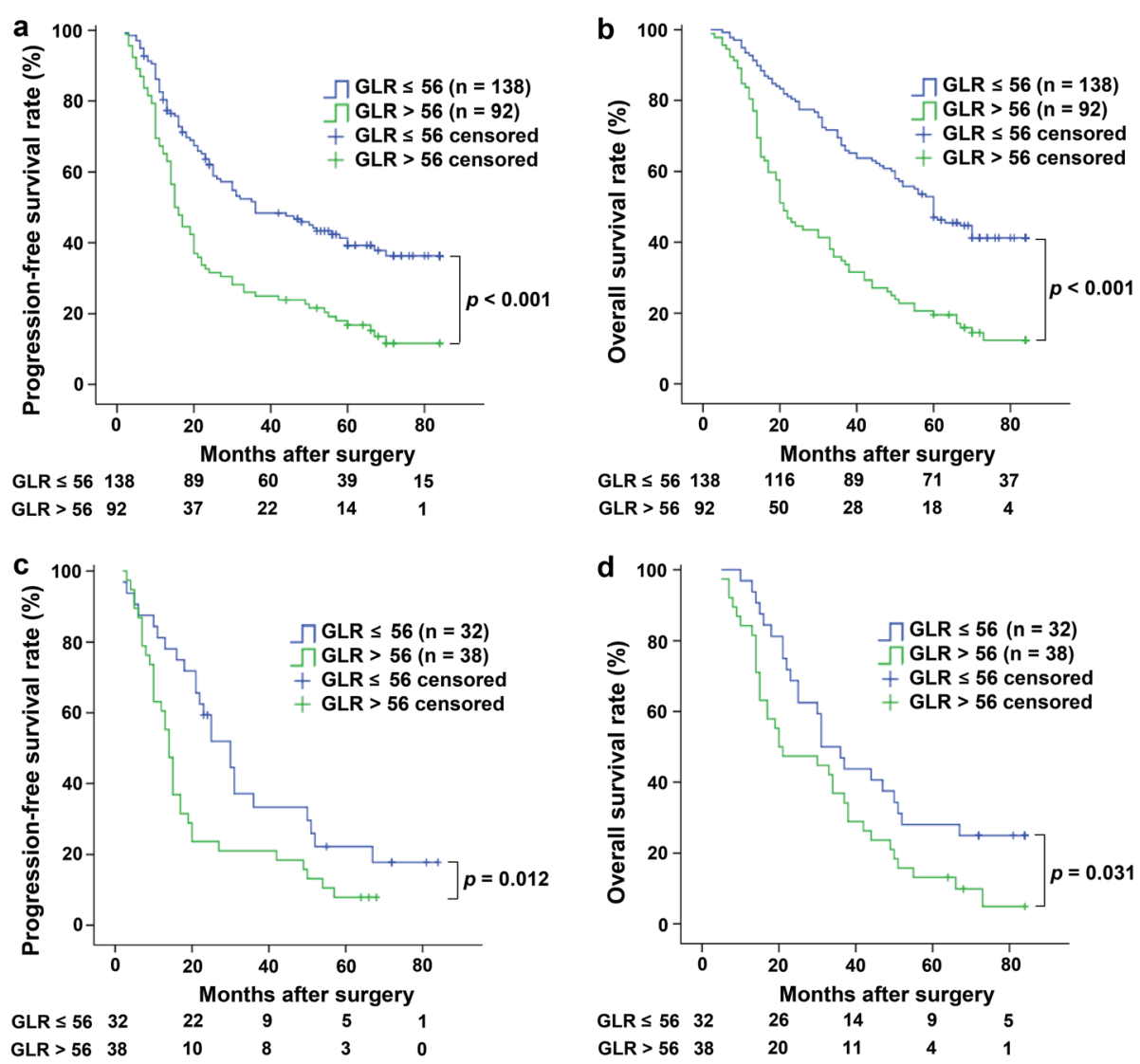

Fig. 3 Kaplan-Meier analysis showed the relationship between GLR and the prognosis of HCC patients. The GLR $\leq 56.0$ group $(n=138)$ had higher OS and PFS rates than the GLR $>56.0$ group $(n=92)$ of HCC patients (both $p<0.001)(\mathbf{a}, \mathbf{b})$. In the M1 group of patients $(n=70)$, the GLR $\leq 56.0$ group $(n=32)$ had higher OS $(p=0.012)$ and PFS $(p=0.031)$ rates than the GLR $>56.0$ group $(n=38)$ of HCC patients $(\mathbf{c}, \mathbf{d})$

determine the prognosis of liver cancer patients treated differently [34], while a high level of AFP may affect the biological behavior of liver cancer, such as invasion, postoperative metastasis and recurrence, and prognosis. Compared with patients with AFP $>20 \mathrm{ng} / \mathrm{ml}$, patients with AFP $\leq 20 \mathrm{ng} / \mathrm{ml}$ had a relatively better survival rate and prognosis [36].

\section{Conclusion}

In clinical practice, the simple, reliable, low-cost and noninvasive serological indicators have significant value in the prediction of risk of MVI occurrence and postoperative prognosis for HCC patients. Elevated GLR level may result in MVI in liver cancer patients, which mechanism is complicated, thus, further researches to clarify it are in need. In conclusion, it is speculated that GLR may serve as an effective factor to predict the risk of MVI and prognosis of HCC patients. However, this is an retrospective study based on limited data from a single agency, and the selection bias cannot be avoided, thus, larger-scale and multi-center studies are in need, prospective studies are even better, in order to illuminate GLR's underlying influence in liver cancer, and provide a basis for planning individual precise intervention therapy and improving prognosis for HCC patients.

\section{Abbreviations}

AFP: Alpha-fetoprotein; ALP: Alkaline phosphatase; ALT: Alanine aminotransferase; AST: Aspartate aminotransferase; AUC: Area under the ROC curve; Cl: Confidence interval; DBIL: Direct bilirubin; GGT: Gamma-glutamyl transpeptidase; GLR: Gamma-glutamyl transpeptidase to lymphocyte count ratio; HBsAg: Hepatitis B surface antigen; HCC: Hepatocellular carcinoma; HR: Hazard ratio; LYMPH: Lymphocyte count; MVI: Microvascular invasion; NEUT: Neutrophil cell count; OS: Overall survival; PFS: Progression-free survival; ROC: Receiver operating characteristic; TBIL: Total bilirubin; WBC: White blood cell

\section{Acknowledgments}

The authors would like to offer special thanks to all clinical staff who worked in the Hepatobiliary and Pancreatic Surgery Laboratory at the Affiliated Hospital of Guilin Medical University.

\section{Authors' contributions}

WL designed the research; HZ, YZ1 (Yu Zhou), YL, WQ, YZ2 (Yunhua Zi), YL, $X Q$ and $H X$ collected the data; $W L$ and $H Z$ performed the data analysis and developed the model; $Y Z 1$ and $Y L$ wrote the first draft of the manuscript; and $H Z, W L$ and $Z H$ critically edited and reviewed the final draft of the 
manuscript; $\mathrm{HZ}, W Q$ and $Y Z 2$ commented on and critically revised the manuscript:; $Y L, X Q$ and $H X$ contributed to the study design, the primary analysis, and manuscript preparation. All the authors contributed to the conception of the study and approved the final manuscript.

\section{Funding}

This work was supported in part by the National Key Sci-Tech Special Project of China (No. 2018ZX10302207), the Science and Technology Planning Project of Guilin (No. 20190218-1), and the students' platform for innovation and entrepreneurship training program (No. 201810601001).

\section{Availability of data and materials}

The datasets used and analyzed in the current study are available from the corresponding author upon reasonable request.

\section{Ethics approval and consent to participate}

The study ethics approval was granted from the local ethical committee of the Affiliated Hospital of Guilin Medical University, and the study was performed in accordance with the principles of the Declaration of Helsinki. Written informed consent was obtained from all patients, including the patient who died during the study.

\section{Consent for publication}

Not applicable.

\section{Competing interests}

The authors declare that they have no competing of interests.

\section{Author details}

'Laboratory of Hepatobiliary and Pancreatic Surgery, Affiliated Hospital of Guilin Medical University, Guilin 541001, Guangxi, People's Republic of China. ${ }^{2}$ Second clinical medical college, Guangxi Medical University, Nanning 530021, Guangxi, People's Republic of China. ${ }^{3}$ Department of Pathology, Guilin Medical University, Guilin 541001, Guangxi, People's Republic of China.

Received: 28 November 2019 Accepted: 11 February 2020

Published online: 18 February 2020

\section{References}

1. Bray F, Ferlay J, Soerjomataram I, Siegel RL, Torre LA, Jemal A. Global cancer statistics 2018: GLOBOCAN estimates of incidence and mortality worldwide for 36 cancers in 185 countries. CA Cancer J Clin. 2018;68(6):394-424.

2. Feng RM, Zong YN, Cao SM, Xu RH. Current cancer situation in China: good or bad news from the 2018 global Cancer statistics? Cancer Commun (Lond). 2019;39(1):22.

3. Mehta N, Heimbach J, Harnois DM, Sapisochin G, Dodge JL, Lee D, et al. Validation of a risk estimation of tumor recurrence after transplant (RETREAT) score for hepatocellular carcinoma recurrence after liver transplant. JAMA Oncol. 2017;3(4):493-500.

4. Cong WM, Bu H, Chen J, Dong H, Zhu YY, Feng LH, et al. Practice guidelines for the pathological diagnosis of primary liver cancer: 2015 update. World J Gastroenterol. 2016;22(42):9279-87.

5. Roayaie S, Blume IN, Thung SN, Guido M, Fiel Ml, Hiotis S, et al. A system of classifying microvascular invasion to predict outcome after resection in patients with hepatocellular carcinoma. Gastroenterology. 2009;137(3):850-5.

6. Eguchi S, Takatsuki M, Hidaka M, Soyama A, Tomonaga T, Muraoka I, et al. Predictor for histological microvascular invasion of hepatocellular carcinoma: a lesson from 229 consecutive cases of curative liver resection. World J Surg. 2010;34(5):1034-8.

7. Wang Q, Xia D, Bai W, Wang E, Sun J, Huang M, et al. Development of a prognostic score for recommended TACE candidates with hepatocellular carcinoma: a multicentre observational study. J Hepatol. 2019;70(5):893-903.

8. Shen L, Zeng Q, Guo P, Huang J, Li C, Pan T, et al. Dynamically prognosticating patients with hepatocellular carcinoma through survival paths mapping based on time-series data. Nat Commun. 2018;9(1):2230.

9. Bottazzi B, Riboli E, Mantovani A. Aging, inflammation and cancer. Semin Immunol. 2018;40:74-82.
10. Comen EA, Bowman RL, Kleppe M. Underlying causes and therapeutic targeting of the inflammatory tumor microenvironment. Front Cell Dev Biol. 2018;6:56.

11. Zhou B, Zhan C, Wu J, Liu J, Zhou J, Zheng S. Prognostic significance of preoperative gamma-glutamyltransferase to lymphocyte ratio index in nonfunctional pancreatic neuroendocrine tumors after curative resection. Sci Rep. 2017;7(1):13372

12. Sumie S, Nakashima O, Okuda K, Kuromatsu R, Kawaguchi A, Nakano M, et al. The significance of classifying microvascular invasion in patients with hepatocellular carcinoma. Ann Surg Oncol. 2014;21(3):1002-9.

13. Zhu PP, Yuan SG, Liao Y, Qin LL, Liao WJ. High level of intercellular adhesion molecule-1 affects prognosis of patients with hepatocellular carcinoma. World J Gastroenterol. 2015:21(23):7254-63.

14. Halazun KJ, Najjar M, Abdelmessih RM, Samstein B, Griesemer AD, Guarrera $\mathrm{JV}$, et al. Recurrence after liver transplantation for hepatocellular carcinoma: a new MORAL to the story. Ann Surg. 2017;265(3):557-64.

15. Qian BZ. Inflammation fires up cancer metastasis. Semin Cancer Biol. 2017; 47:170-6.

16. Chiba T, Marusawa H, Ushijima T. Inflammation-associated cancer development in digestive organs: mechanisms and roles for genetic and epigenetic modulation. Gastroenterology. 2012;143:550-63.

17. Coussens LM, Werb Z. Inflammation and cancer. Nature. 2002;420(6917): 860-7.

18. Coffelt SB, de Visser KE. Cancer: inflammation lights the way to metastasis. Nature. 2014;507(7490):48-9.

19. Sarvaiya PJ, Guo D, Ulasov I, Gabikian P, Lesniak MS. Chemokines in tumor progression and metastasis. Oncotarget. 2013;4(12):2171-85.

20. Greten FR, Grivennikov SI. Inflammation and cancer: triggers, mechanisms, and consequences. Immunity. 2019;51(1):27-41.

21. Dolgin E. Anti-inflammatory drug cuts risk of heart disease - and cancer. Nat Rev Drug Discov. 2017;16(10):665-7.

22. Mann J, Reeves HL, Feldstein AE. Liquid biopsy for liver diseases. Gut. 2018; 67(12):2204-12.

23. Fung J, Cheung KS, Wong DK, Mak LY, To WP, Seto WK, et al. Long-term outcomes and predictive scores for hepatocellular carcinoma and hepatitis B surface antigen seroclearance after hepatitis B e-antigen seroclearance. Hepatology. 2018;68(2):462-72.

24. Qu C, Wang Y, Wang P, Chen K, Wang M, Zeng H, et al. Detection of earlystage hepatocellular carcinoma in asymptomatic HBsAg-seropositive individuals by liquid biopsy. Proc Natl Acad Sci U S A. 2019;116(13):6308-12.

25. Ikeda Y, Taniguchi N. Gene expression of gamma-glutamyltranspeptidase. Methods Enzymol. 2005:401:408-25.

26. Carr Bl, Pancoska P, Branch RA. Low alpha-fetoprotein hepatocellular carcinoma. J Gastroenterol Hepatol. 2010;25(9):1543-9.

27. Ju MJ, Qiu SJ, Fan J, Zhou J, Gao Q, Cai MY, et al. Preoperative serum gamma-glutamyl transferase to alanine aminotransferase ratio is a convenient prognostic marker for child-Pugh a hepatocellular carcinoma after operation. J Gastroenterol. 2009;44(6):635-42.

28. Zhao WC, Fan LF, Yang N, Zhang HB, Chen BD, Yang GS. Preoperative predictors of microvascular invasion in multinodular hepatocellular carcinoma. Eur J Surg Oncol. 2013:39(8):858-64.

29. Ma H, Zhang L, Tang B, Wang Y, Chen R, Zhang B, et al. $\gamma^{-}$ Glutamyltranspeptidase is a prognostic marker of survival and recurrence in radiofrequency-ablation treatment of hepatocellular carcinoma. Ann Surg Oncol. 2014:21(9):3084-9.

30. Zhang JB, Chen Y, Zhang B, Xie X, Zhang L, Ge N, et al. Prognostic significance of serum gamma-glutamyl transferase in patients with intermediate hepatocellular carcinoma treated with transcatheter arterial chemoembolization. Eur J Gastroenterol Hepatol. 2011;23(9): 787-93.

31. Mantovani A, Allavena P, Sica A, Balkwill F. Cancer-related inflammation. Nature. 2008;454(7203):436-44.

32. Rosenberg SA. Progress in human tumour immunology and immunotherapy. Nature. 2001;411(6835):380-4.

33. Ding PR, An X, Zhang RX, Fang YJ, Li LR, Chen G, et al. Elevated preoperative neutrophil to lymphocyte ratio predicts risk of recurrence following curative resection for stage IIA colon cancer. Int J Color Dis. 2010; 25(12):1427-33.

34. Liu PH, Hsu CY, Hsia CY, Lee YH, Su CW, Huang YH, et al. Prognosis of hepatocellular carcinoma: assessment of eleven staging systems. J Hepatol. 2016;64(3):601-8. 
35. Rodríguez-Perálvarez M, Luong TV, Andreana L, Meyer T, Dhillon AP Burroughs AK. A systematic review of microvascular invasion in hepatocellular carcinoma: diagnostic and prognostic variability. Ann Surg Oncol. 2013:20(1):325-39.

36. Chan SL, Chan AT, Yeo W. Role of alpha-fetoprotein in hepatocellular carcinoma: prognostication, treatment monitoring or both? Future Oncol. 2009:5(6):889-99.

\section{Publisher's Note}

Springer Nature remains neutral with regard to jurisdictional claims in published maps and institutional affiliations.

Ready to submit your research? Choose BMC and benefit from:

- fast, convenient online submission

- thorough peer review by experienced researchers in your field

- rapid publication on acceptance

- support for research data, including large and complex data types

- gold Open Access which fosters wider collaboration and increased citations

- maximum visibility for your research: over $100 \mathrm{M}$ website views per year

At $\mathrm{BMC}$, research is always in progress. 